University of Nebraska - Lincoln

DigitalCommons@University of Nebraska - Lincoln

Roger Kirby Publications

Research Papers in Physics and Astronomy

November 1991

\title{
Studies of domain dynamics in amorphous Dy/Fe multilayers
}

Roger D. Kirby

University of Nebraska-Lincoln, rkirby1@unl.edu

J.X. Shen

University of Nebraska - Lincoln

Z.S. Shan

University of Nebraska - Lincoln

David J. Sellmyer

University of Nebraska-Lincoln, dsellmyer@unl.edu

Follow this and additional works at: https://digitalcommons.unl.edu/physics_kirby

Part of the Physics Commons

Kirby, Roger D.; Shen, J.X.; Shan, Z.S.; and Sellmyer, David J., "Studies of domain dynamics in amorphous Dy/Fe multilayers" (1991). Roger Kirby Publications. 37.

https://digitalcommons.unl.edu/physics_kirby/37

This Article is brought to you for free and open access by the Research Papers in Physics and Astronomy at DigitalCommons@University of Nebraska - Lincoln. It has been accepted for inclusion in Roger Kirby Publications by an authorized administrator of DigitalCommons@University of Nebraska - Lincoln. 


\title{
Studies of domain dynamics in amorphous Dy/Fe multilayers
}

\author{
Roger D. Kirby, J. X. Shen, Z. S. Shan, and D. J. Sellmyer
}

Behlen Laboratory of Physics and Center for Materials Research and Analysis, University of Nebraska, Lincoln. Nebraska 68588-0111

Compositionally-modulated $\mathrm{Dy} / \mathrm{Fe}$ films were prepared by dc magnetron sputtering onto glass substrates. For this series of samples, the Dy thickness was fixed at $5 \AA$, while the Fe thickness was varied from 4 to $6 \AA$. To study the magnetization reversal, the samples were first saturated. The magnetic field was then reversed to a value near the coercive field, and the Kerr rotation was measured as a function of time after reversal. We interpret our results in terms of the model of domain reversal first described by Fatuzzo for ferroelectrics. For some Fe thicknesses, we found that the reversal process was dominated by domain nucleation, while for other thicknesses it was dominated by domain wall motion. The observed behavior is discussed in terms of the magnetic and structural properties of the films.

\section{INTRODUCTION}

We report a study of the magnetization reversal process in amorphous Dy/Fe multilayer samples. The individual layer thicknesses in the samples investigated here are typically $5 \AA$ and hence in the regime where the samples are amorphous and have perpendicular magnetic anisotropy, ${ }^{1}$ so that these materials are potentially useful as magneto-optic storage media. We will see that our samples show two qualitatively different kinds of magnetization reversal behavior, each of which can be understood in terms of existing models of magnetization reversal.

\section{EXPERIMENT}

The $\mathrm{Dy} / \mathrm{Fe}$ samples were prepared by two-gun dc magnetron sputtering onto glass substrates at room temperature. The base pressure of the sputtering chamber was $2 \times 10^{-7}$ Torr. For the series of samples discussed here, the Dy thickness was held at $5 \AA$, while the Fe thickness was varied from 4.5 to $6.0 \AA$. All samples in this sequence were prepared during the same vacuum run under nominally identical conditions. Large-angle $x$-ray diffraction measurements showed only broad structure, with no evidence of crystalline phases. Small-angle $\mathrm{x}$-ray diffraction measurements showed only a weak peak near a $d$ spacing of $10 \AA$, indicating that the compositional modulation is approximately sinusoidal in character. Thus these samples can be properly described as amorphous compositionallymodulated films. ${ }^{1}$ These materials are nearly magnetically compensated, with the Dy subnetwork magnetization being generally antiparallel to that of $\mathrm{Fe}$. Below the compensation temperature the Dy moments dominate, while above the compensation temperature the $\mathrm{Fe}$ moments dominate. ${ }^{2}$ The compensation temperature of the $\mathrm{Dy}(5 \AA) / \mathrm{Fe}(6 \AA)$ sample is below room temperature, while the compensation temperatures of the other three samples are all above room temperature.

Polar Kerr rotation measurements were carried out at room temperature with light of wavelength $632.8 \mathrm{~nm}$ using apparatus described elsewhere. ${ }^{3}$ In order to study magnetization reversal in these materials, the samples were first magnetically saturated using a field of $15 \mathrm{kOe}$. The mag- netic field was then reversed to near the coercive field, and the Kerr rotation was monitored as a function of time after reversal.

\section{RESULTS AND DISCUSSION}

Figure 1 shows the Kerr rotation $\left(\theta_{K}\right)$ as a function of time after magnetic field reversal for $\operatorname{Dy}(5 \AA) / \mathrm{Fe}(4.5 \AA)$ for a number of different field values. In this figure, the $\theta_{K}=0$ line corresponds to complete magnetization reversal. It should be noted that for this sample complete reversal only occurs for fields greater than or equal to the saturation field.

Figure 2 shows the magnetization reversal time dependence for $\operatorname{Dy}(5 \AA) / \mathrm{Fe}(5 \AA)$ at several field values. The time dependence is qualitatively different from that shown in Fig. 1. Note that if the experiment is continued long enough, complete reversal occurs even for fields considerably smaller than the coercive field.

To interpret these results, we consider the model of Fatuzzo, who considered polarization reversal in ferroelectrics. ${ }^{4}$ Labrune et al. ${ }^{5}$ have used this model to describe magnetization reversal in other rare-earth/ transition-metal films, and Pommier et al. ${ }^{6}$ have applied it to ultrathin Co films. This model assumes that the sample contains domain reversal nucleation sites which reverse by thermal activation at a rate proportional to the number of nucleation sites remaining. Once a domain is nucleated, it grows by domain wall motion. The time dependence of the reversal curve depends on the relative magnitudes of the nucleation rate and the domain wall velocity. For our thinfilm samples, we assume that the domains extend through the thickness of the sample. This model then predicts that the fractional area not yet reversed has the functional form:

$$
\begin{aligned}
A(t)= & \exp \left\{-2 k^{2}+2 k^{2} R t+2 k-(k R t)^{2}-2 k R t\right. \\
& \left.-R t+2 k^{2}\left(1-k^{-1}\right) \exp (-R t)\right\} .
\end{aligned}
$$

Here, $R$ is the rate of domain nucleation according to the equation $N_{R}=N_{0}(1-\exp (-R t))$, where $N_{0}$ is the total number of nucleation sites and $N_{R}$ is the number of sites that have reversed at time $t . k$ is a parameter related 


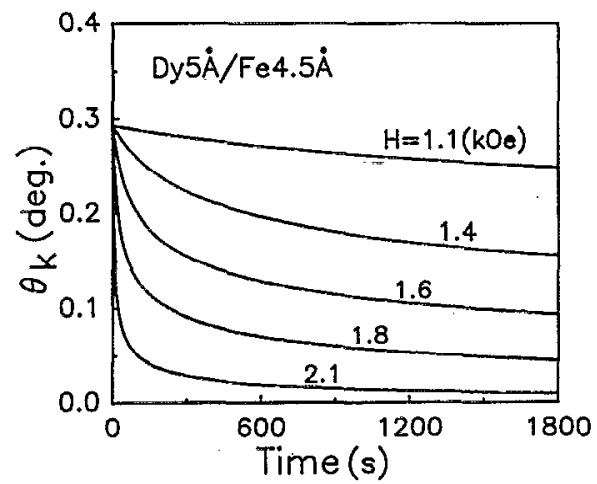

FIG. 1. Kerr rotation as a function of time after magnetization reversal for $\mathrm{Dy}(5 \AA) / \mathrm{Fe}(4.5 \AA)$ for several different values of the reversing field. The coercive field of this sample is $1.7 \mathrm{kOe}$.

to the average velocity of domain wall motion, with $k$ $=V_{W} / R r_{c}$, where $V_{W}$ is the domain wall velocity and $r_{c}$ is the initial radius of the domains.

For our purposes, there are two limiting cases. If $R$ is small and $V_{W}$ is large (so that $k$ is also large), then nucleation is an infrequent event, but once it occurs, the domains grow rapidly. This case is appropriate for Dy $(5 \AA) /$ $\mathrm{Fe}(5 \AA)$. Figure 3 shows the fits of the Fatuzzo model to the time dependence for this sample. The agreement is quite remarkable considering the simplicity of the model. The most obvious approximations in this model are the assumption of a single rate of nucleation (rather than a distribution of rates) and a single domain wall velocity. The quality of the fits suggests that both of these assumptions are true to a reasonable approximation. The values of $R$ and $k$ obtained from the fits are summarized in Fig. 4. It should be noted that the experimental curves in Fig. 3 can also be scaled (by plotling $\theta_{K}$ versus $t / t_{50}$, where $t_{50}$ is the time required for the Kerr rotation to decay by $50 \%$ ) as shown by Labrune et al., so that all curves collapse quite closely onto one curve.

In the $k=0$ limit, magnetization reversal in the Fatuzzo model occurs only by nucleation, so that a purely exponential time decay is expected. The general form of the magnetization reversal data for $\operatorname{Dy}(5 \AA) / \mathrm{Fe}(4.5 \AA)$ (Fig.

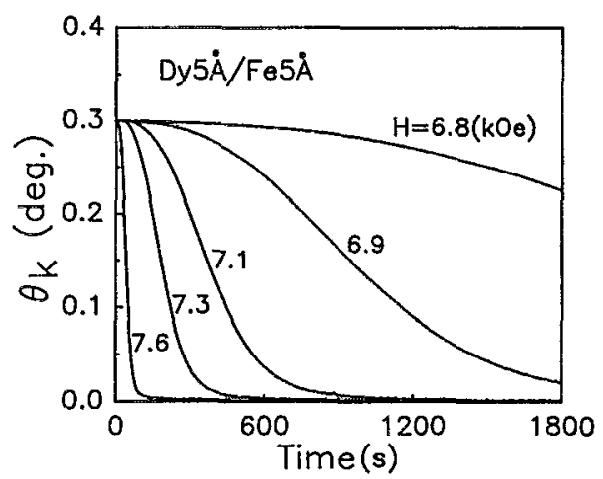

FIG. 2. Kerr rotation as a function of time after magnetization reversal for $\operatorname{Dy}(5 \AA) / \mathrm{Fe}(5 \AA)$ for several different values of the reversing field. The coercive field of this sample is $7.4 \mathrm{kOe}$.

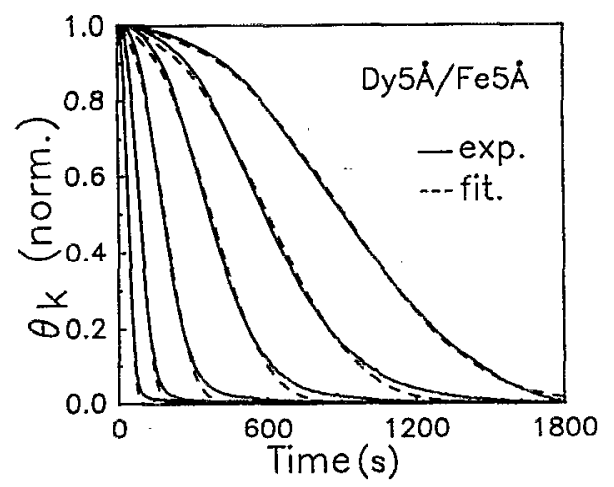

FIG. 3. Kerr rotation as a function of time for $\mathrm{Dy}(5 \AA) / \mathrm{Fe}(5 \AA)$. The solid lines are the measured time dependences, while the dashed lines are nonlinear least-squares fits to Eq. (1).

1) suggests that it falls in this limit. We find however that the magnetization reversal data in Fig. 1 cannot be well described by the Fatuzzo model. The rate of reversal in the long time limit is simply too small for a good fit to be achieved. This suggests that there is a fairly broad distribution in thermal activation energies for domain nucleation. Further evidence for this is given in Fig. 5, which shows $\theta_{K}$ as a function of $\ln (t)$ for large $t$. In this case, we find approximately linear behavior as predicted by the model of Street and Woolley for a system with a broad distribution of domain-reversal activation energies. ${ }^{7}$ As expected, there is rough agreement between the field dependence of the slope of the $\theta_{K}$ versus $\ln (t)$ curves of Fig. 5 and the switching field distribution obtained from the derivative of the hysteresis loop. These two curves are shown in Fig. 6.

It should be noted that $\mathrm{Dy}(5 \AA) / \mathrm{Fe}(5.5 \AA)$ shows the same qualitative magnetization reversal behavior as $\mathrm{Dy}(5$ $\AA) / \mathrm{Fe}(5 \AA)$, while $\mathrm{Dy}(5 \AA) / \mathrm{Fe}(6 \AA)$ behaves similarly to $\mathrm{Dy}(5 \AA) / \mathrm{Fe}(4.5 \AA)$. All of the samples in the sequence were prepared in the same sputtering run under presumably identical conditions. However, we have not yet studied the thickness dependence systematically enough to determine if the two different behaviors are due to a strong and nonmonotonic dependence on Fe thickness, or due to subtle, and as of yet unidentified, differences in nanostruc-

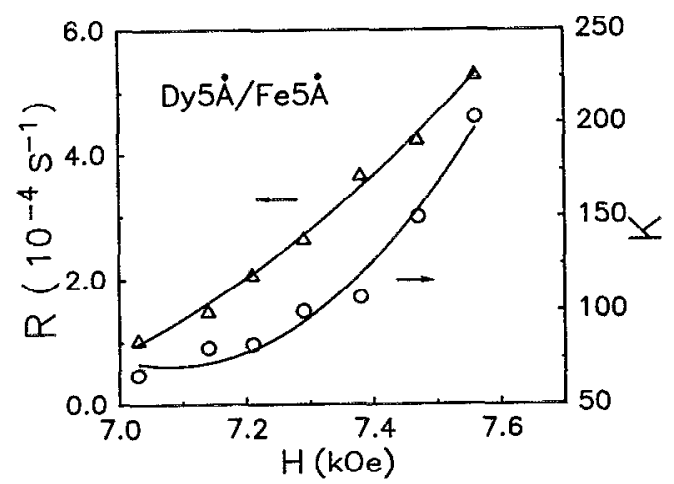

FIG. 4. Magnetic-field dependences of fitting parameters. Triangles: $\mathrm{Nu}$ cleation rate $R$; circles: parameter $k$. The lines are guides to the eye. 


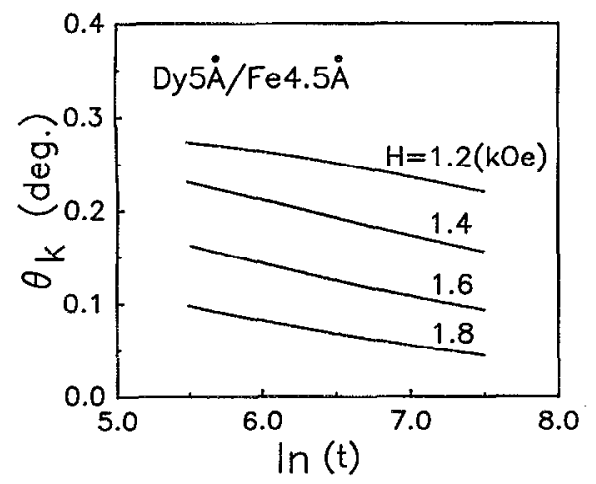

FIG. 5. Kerr rotation vs $\ln (t)$ for $\operatorname{Dy}(5 \AA) / F e(4.5 \AA)$, where $t$ is the time after reversal of the applied magnetic field. The applied fields are indicated. Note the approximately linear behavior for long times.

ture. One intriguing possibility is that the magnetization reversal behavior can be modified in a controlled manner by simply changing the $\mathrm{Fe}$ layer thickness. For example, magnetization reversal in the $\mathrm{Fe}(5 \AA)$ and $\mathrm{Fe}(5.5 \AA)$ samples is described quite well by the Fatuzzo model. These samples also correspond to approximately two monolayers

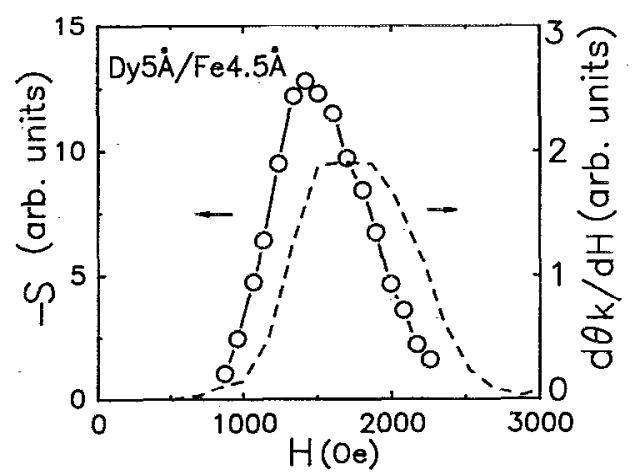

FIG. 6. Slope of the $\theta_{K}$ vs $\ln (t)$ curves of Fig. 5 as a function of applied (reversed) magnetic field (open circles) and the switching field distribution (dashed line) obtained from the derivative of the hysteresis loop for $\mathrm{Dy}(5 \AA) / \mathrm{Fe}(4.5 \AA)$ at room temperature. of Fe. This may indicate that an integral number of $\mathrm{Fe}$ layers results in a more uniform or flatter layer structure in these samples, and hence to a simpler magnetization reversal behavior. We have also prepared a second sequence of samples of the form $\operatorname{Dy}(X \AA) / \mathrm{Fe}(X \AA)$. While we have not yet completed measurements on these samples, the $X$ $=5.5 \AA$ sample showed behavior similar to that of Fig. 2, lending some credence to this idea.

\section{SUMMARY AND CONCLUSIONS}

We have studied magnetization reversal in amorphous $\mathrm{Dy} / \mathrm{Fe}$ multilayers at room temperature and found two distinctly different kinds of behavior. Samples Dy $(5 \AA) /$ $\mathrm{Fe}(5 \AA)$ and $\mathrm{Dy}(5 \AA) / \mathrm{Fe}(5.5 \AA)$ showed reversal behavior which could be quite well described by the Fatuzzo model, suggesting a relatively narrow distribution of domain nucleation activation energies. Magnetization reversal in samples $\mathrm{Dy}(5 \AA) / \mathrm{Fe}(4.5 \AA)$ ) and $\mathrm{Dy}(5 \AA) / \mathrm{Fe}(6 \AA)$ could not be described by the Fatuzzo model, and their long-time behavior indicated a broad distribution of domain nucleation activation energies. Further study of this system will include temperature dependence measurements to more completely characterize the reversal behavior and new samples will be investigated to determine if an integral number of $\mathrm{Fe}$ layers does indeed result in a more uniform layer structure and hence simpler reversal behavior.

\section{ACKNOWLEDGMENTS}

We gratefully acknowledge financial support from the Research Corporation and from the National Science Foundation under Grant DMR-8918889.

${ }^{\mathrm{I}}$ Z. S. Shan and D. J. Sellmyer, Phys. Rev. B 42, 10433 (1990).

${ }^{2}$ See K. M. Moorjani and J. M. D. Coey, Magnetic Glasses (Elsevier, New York, 1989).

${ }^{3}$ J. X. Shen, R. D. Kirby, and D. J. Sellmyer, J. Magn. Magn. Mater. 81, 107 (1989).

${ }^{4}$ E. Fatuzzo, Phys. Rev. 127, 1999 (1962).

${ }^{5}$ M. Labrune, S. Andrieu, F. Rio, and P. Bernstein, J. Magn. Magn. Mater. 80, 211 (1989).

${ }^{6} \mathrm{~J}$. Pommier, P. Meyer, G. Pénissard, J. Ferré, P. Bruno, and D. Renard, Phys. Rev. Lett. 65, 2054 (1990).

${ }^{7}$ R. Street and J. C. Woolley, Proc. Phys. Soc. A 62, 562 (1949). 